\title{
Spectacle Independence in Patients with Prior Radial Keratotomy Following Cataract Surgery: A Case Series
}

\author{
Smita Agarwal ${ }^{1,2}$ \\ Erin Thornell $\mathbb{D}^{2}$ \\ 'University of Wollongong, Wollongong, \\ Australia; ${ }^{2}$ Wollongong Eye Specialist, \\ Wollongong, Australia
}

This article was published in the following Dove Press journal: International Medical Case Reports Journal

Purpose: To assess if post-keratorefractive patients can achieve spectacle independence following cataract surgery with currently available presbyopia correcting intraocular lenses (IOLs). Patients and Methods: Retrospective case series of unilateral implantation of the IC-8 IOL in two patients with history of bilateral myopic radial keratotomy (RK) and one patient with history of bilateral myopic RK and astigmatic keratotomy (AK), and bilateral implantation of AT Lisa 939M multifocal IOLs in one patient with previous history of RK and laser in situ keratomileusis (LASIK).

Results: Good uncorrected distance, intermediate and near visual acuity (VA) was achieved for most patients. Stereopsis and contrast sensitivity (CS) were maintained in patients that received the IC-8 IOLs, and two patients achieved full spectacle independence. The patient that received the trifocal lenses expressed satisfaction with their vision, but required long distance correction and experienced reduced CS.

Conclusion: The IC-8 and AT Lisa 939M IOLs both offer satisfactory results and reduced spectacle dependence in post-keratorefractive patients. However, it is recommended to properly counsel visual outcomes and patient expectations before commencing surgery.

Keywords: post-refractive, multifocal, cataract, presbyopia correcting

\section{Introduction}

Radial keratotomy (RK) and laser in situ keratomileusis (LASIK) were highly popular in the 1980s and 1990s to correct refractive error. As the population ages, patients with history of RK and LASIK are increasingly presenting with cataracts. Such patients frequently desire spectacle independence, but present multiple technical challenges for the surgeon. LASIK and RK can result in corneal aberrations and progressive corneal flattening that can cause hyperopic shift, and in the case of LASIK, inconsistencies between the anterior and posterior corneal curvature. This complicates lens power calculations, making postoperative outcomes unpredictable.

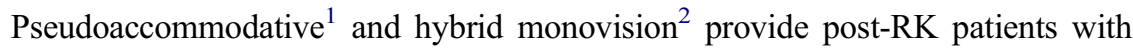
depth of focus, but do not correct corneal aberrations that can affect patient satisfaction. Multifocal intraocular lenses (IOLs) can provide good vision at all distances, ${ }^{3}$ but there is a high incidence of visual symptoms such as glare and haloes due to the multiple refractive zones of the lens that emphasize the effect of corneal aberrations. ${ }^{4,5}$

The IC-8 IOL combines pinhole technology, through the use of an opaque mask composed of polyvinylidene difluoride and carbon nanoparticles with a central $1.36 \mathrm{~mm}$ aperture, with an aspheric hydrophobic acrylic monofocal lens platform.
Correspondence: Smita Agarwal

13 Market Street, Wollongong, NSW

2500 , Australia

Tel +6I $24423671 I$

Fax +6I $2426305 \mid I$

Email smitaagarwal@hotmail.com 
The pinhole effect may attenuate the impact of corneal aberrations, while providing continual extended depth of focus. The AT Lisa 939M IOL is an aspheric trifocal lens. Aspheric lenses reduce the amount of induced higher order aberrations (HOAs), particularly spherical aberration (SA), and result in higher postoperative mesopic VA, contrast sensitivity (CS) and optical transfer function compared to spheric lenses. ${ }^{6-9}$

This case series reports two cases of cataract surgery in patients with previous history of RK and one case with previous RK and astigmatic keratotomy (AK), where an IC-8 IOL was implanted into the non-dominant eye and an aspheric IOL was implanted into the dominant eye, and one case of bilateral implantation of trifocal AT Lisa 939M IOLs in a patient with previous history of RK and LASIK.

\section{Patients and Methods}

Routine uncomplicated phacoemulsification was performed by a single surgeon (SA) bilaterally on two patients with previous history of bilateral myopic RK, one patient with previous history of RK and AK, and one patient with previous history of myopic RK and LASIK. Each post-RK patient had between 8 and 16 incisions, however pre-keratorefractive refractions and other information regarding the procedures were unavailable as they were performed up to 30 years ago by a different surgeon. Lens power was calculated using the Barrett True-K calculator, available on the Asia-Pacific Association of Cataract and Refractive Surgeons website (calc.apacrs.org/barrett_universal2015/), using a target refraction of $-0.75 \mathrm{D}$ for eyes to receive the IC- 8 IOL and between 0 $\mathrm{D}$ and $\pm 0.5 \mathrm{D}$ for the eyes to receive the standard monofocal and trifocal IOLs. Following surgery, the patients were instructed to commence ofloxacin (Allergan; Ocuflox; Dublin, Ireland) and prednisolone acetate/phenylephrine hydrochloride (Allergan; Prednefrin forte; Dublin, Ireland) eye drops, applying one drop every 2 hrs until bed time. From the following day, the patients were advised to continue using ofloxacin and prednisolone acetate/phenylephrine hydrochloride eye drops applying one drop qid for 2 weeks and 3-4 weeks respectively, and to commence using ketorolac (Allergan; Acular; Dublin, Ireland) eye drops applying one drop qid for 3 days. At 1 and 6 months postoperatively, uncorrected distance (UDVA; equivalent to 6 metres), intermediate (UIVA; equivalent to 1 metre) and near (UNVA; equivalent to $40 \mathrm{~cm}$ ) $\mathrm{VA}$, manifest refraction and uncorrected stereoacuity were measured. Stereoacuity was measured using the Stereo Fly Test at $40 \mathrm{~cm}$. Uncorrected contrast sensitivity function (CSF) was measured under mesopic conditions using a sinus chart, and modulation transfer function (MTF) was measured using an iTrace (Tracey Technologies; Houston, USA) autorefractor and a $2 \mathrm{~mm}$ pupil size. Mean values were compared using a paired $T$-test assuming $\mathrm{P}<0.05$ was statistically significant. A questionnaire, adapted from the Pseudophakic Dysphotopsia Questionnaire published by Kinard et $\mathrm{al}^{10}$ was also performed to assess the impact of visual artefacts such as glare and haloes, and spectacle dependence. Informed written consent to publish was obtained from all patients prior to commencing postoperative testing. Institutional approval was not required to conduct this study or to publish the results.

\section{Case I}

A 73-year-old patient presented complaining of deterioration of vision in both eyes. The patient had undergone bilateral myopic RK with monovision +30 years previously; the right eye was adjusted for near vision and the left eye was adjusted for distance vision. Preoperative manifest refraction was $-1.25 \mathrm{DS} /-1.5 \mathrm{DC} \times 88^{\circ}$ and $+0.5 \mathrm{DS} /-2.25$ $\mathrm{DC} \times 108^{\circ}$ in the right and left eye respectively, and best corrected distance visual acuity (CDVA) was 20/125 in the right eye and 20/40 in the left eye. Measured total preoperative corneal HOAs were $0.286 \mu \mathrm{m}$ and $0.314 \mu \mathrm{m}$ in the right and left eyes respectively. Coma scores were $0.106 \mu \mathrm{m}$ in the right eye and $0.098 \mu \mathrm{m}$ in the left eye. The patient was found to have bilateral dense cataracts worse in the left eye along with posterior vitreous detachment in the right eye. Corneal topography showed bilateral irregular astigmatism and corneal flattening (Figure 1). The left eye received a +24.5 D AcrySof IQ SN6AT4 toric lens (Alcon; SN6AT4; Texas, USA) and the right eye received a +22.5 D IC-8 (AcuFocus; Irvine, California, USA) lens. At the final visit, refraction was $-1.0 \mathrm{DS}$ in the IC- 8 eye and -0.25 DS/-0.50 DC $\times 110^{\circ}$ in the monofocal toric eye. Uncorrected visual acuities were 20/20, 20/16, and N6 for distance, intermediate and near vision respectively for the IC-8 eye, 20/20, 20/20 and N10 respectively for the monofocal toric eye, and 20/20, 20/16 and N5 respectively for both eyes. Stereoacuity threshold was 200 seconds of arc (sec arc). The patient did not require spectacles and did not report any visual disturbances.

\section{Case 2}

A 65-year-old patient presented complaining of deteriorating vision. The patient had undergone bilateral myopic RK +20 years previously. Preoperative manifest refraction was +2.75 $\mathrm{DS} /-0.75 \mathrm{DC} \times 73^{\circ}$ and $+1.75 \mathrm{DS} /-0.5 \mathrm{DC} \times 150^{\circ}$ in the 

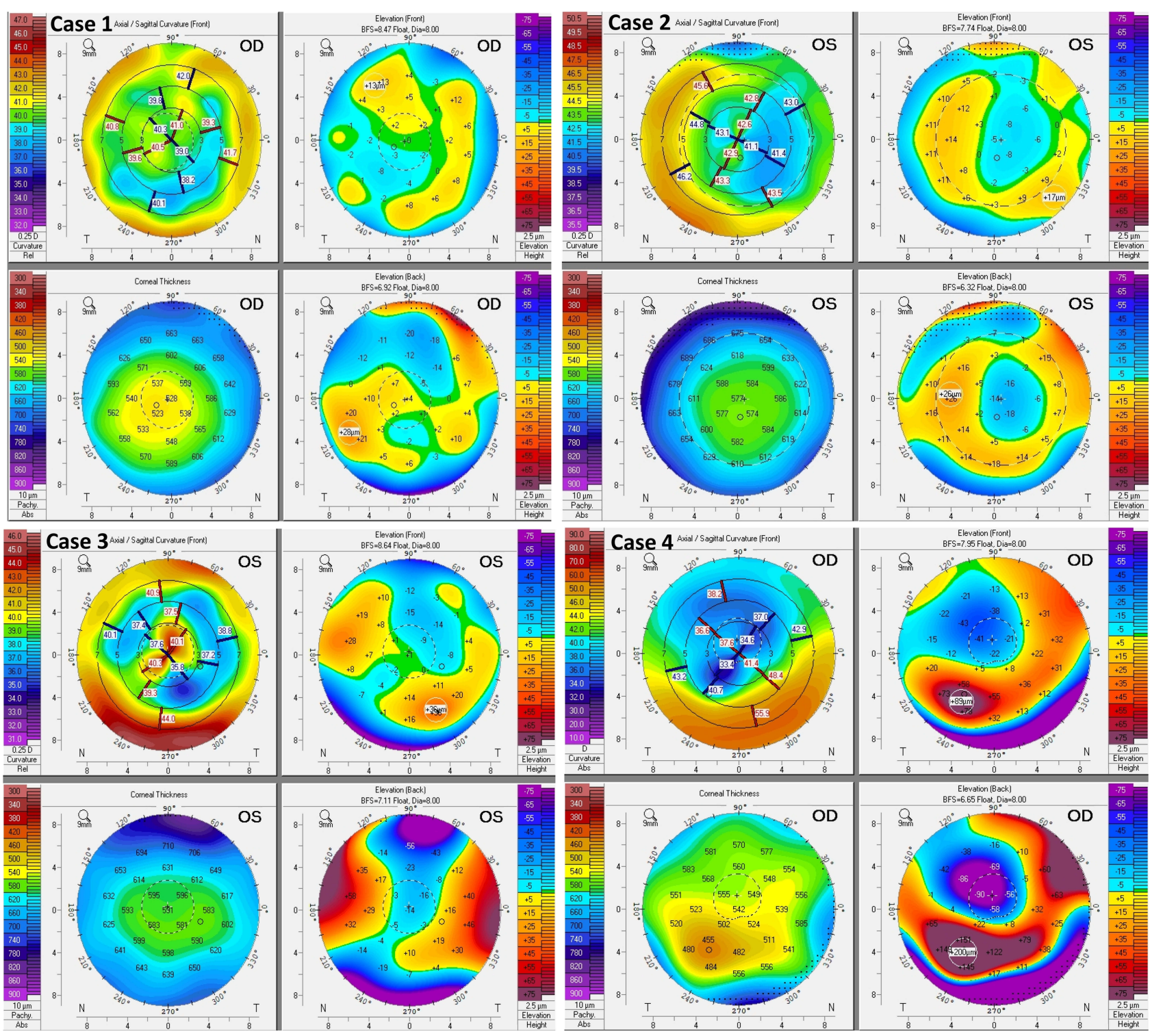

Figure I Preoperative corneal topography of a patient with previous history of bilateral myopic RK with monovision (Case I), of bilateral myopic RK (Case 2), of bilateral RK and AK (Case 3) and of bilateral myopic RK and LASIK (Case 4) showing central irregular corneal flattening.

right and left eye respectively, and CDVA was 20/25 in the right eye and 20/20 in the left eye. Measured total preoperative corneal HOAs were $0.350 \mu \mathrm{m}$ and $0.210 \mu \mathrm{m}$, with coma scores of $0.26 \mu \mathrm{m}$ and $0.16 \mu \mathrm{m}$ in the left and right eyes respectively. Cataracts were identified bilaterally and an epiretinal membrane was noted in the right eye. Corneal topography showed bilateral irregular astigmatism and central corneal flattening (Figure 1). The right eye received a +24.5 D AcrySof IQ SN60WF monofocal lens (Alcon; Texas, USA) and the left eye received a +25.0 D IC- 8 lens. At the final visit, refraction was plano/- $0.50 \mathrm{DC} \times 150^{\circ}$ in the IC-8 eye and plano/-0.75 DC $\times 65^{\circ}$ in the monofocal eye. Uncorrected visual acuities were 20/40, 20/40, and N10 for distance, intermediate and near vision respectively for the IC-8 eye, 20/35, 20/12.5 and N8 for the monofocal eye, and 20/25, 20/12.5 and N6 respectively for both eyes. Stereoacuity threshold was $400 \mathrm{sec}$ arc. The patient did not require spectacles and did not report any visual disturbances.

\section{Case 3}

A 72-year-old patient presented complaining of flashes and floaters in the right eye. The patient had undergone bilateral RK and $\mathrm{AK}+25$ years previously. Preoperative manifest refraction was $+1.0 \mathrm{DS} /-0.25 \mathrm{DC} \times 90^{\circ}$ and $+1.5 \mathrm{DS} /-1.5 \mathrm{DC} \times 112^{\circ}$ in the right and left eye respectively, and BCVA was 20/40 in the right eye and 20/60 in 
the left eye. Measured total preoperative corneal HOAs were $0.503 \mu \mathrm{m}$ and $0.504 \mu \mathrm{m}$, with coma scores of 0.11 $\mu \mathrm{m}$ and $0.21 \mu \mathrm{m}$ in the left and right eyes respectively. The patient was diagnosed with posterior vitreous detachment in the right eye accompanied by vitreous haemorrhage, and early nuclear sclerosis. Corneal topography showed irregular astigmatism and central corneal flattening (Figure 1). Retinopexy was performed in the right eye for a retinal tear, and BCVA was maintained at 20/24 in that eye. Two years following the retinopexy procedure, a +19.5 AcrySof IQ SN6AT4 toric lens was implanted in the right eye, and a +22.0 IC- 8 lens was subsequently implanted in the left eye. At the final visit, refraction was plano/ $-1.75 \mathrm{DC} \times 110^{\circ}$ in the IC- 8 eye and $+0.5 \mathrm{DS}$ in the monofocal eye. Uncorrected visual acuities were 20/ 25, 20/16 and N10 in the IC-8 eye, 20/20, 20/25 and N12 in the monofocal eye and 20/20, 20/16 and N8 with both eyes for distance, intermediate and near vision respectively. Stereoacuity threshold was $>400 \mathrm{sec}$ arc. The patient required spectacles only for reading small print, but did not report any visual disturbances.

Figure 2 shows intraoperative images of the left eye of Case 3, prior to and following implantation of the IC-8 IOL.

\section{Case 4}

A 69-year-old patient presented with increasing hyperopia and astigmatism following bilateral myopic RK and myopic LASIK +25 years previously. Preoperative manifest refraction was +2.5 DS/-1.0 DC x $91^{\circ}$ and +3.25 DS/-3.0

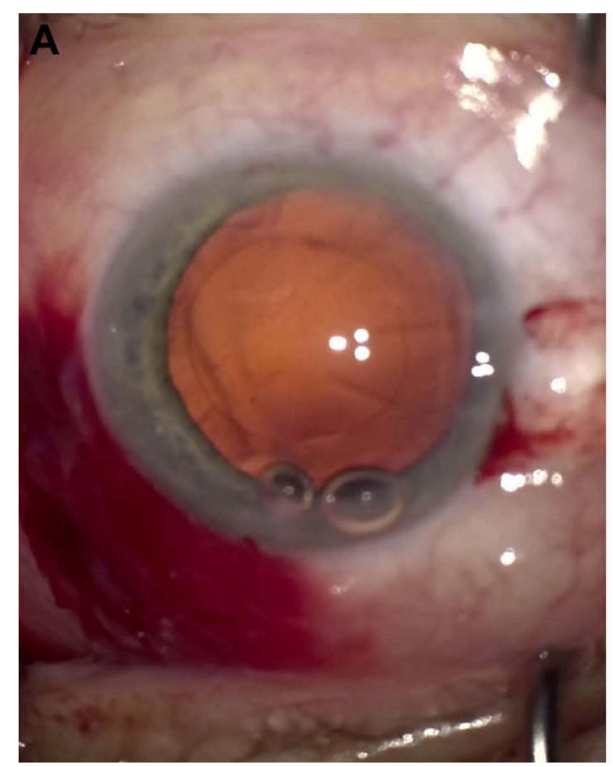

DC $x 112^{\circ}$ in the right and left eye respectively, and BCVA was $20 / 30$ in the right eye and $20 / 25$ in the left eye. Measured total preoperative corneal HOAs were $0.394 \mu \mathrm{m}$ and $0.352 \mu \mathrm{m}$, with coma scores of $0.019 \mu \mathrm{m}$ and $0.154 \mu \mathrm{m}$ in the right and left eyes respectively. Corneal topography showed irregular astigmatism and central corneal flattening (Figure 1). A diffractive aspheric 22.0 cyl +9.0 AT Lisa tritoric 939M IOL (Zeiss; Oberkochen, Germany) was implanted into the right eye, and a sph +21.5 D cyl +9.5 D 939M tritoric AT Lisa IOL was implanted into the left eye. At the final visit, refraction was plano/-3.5 $\mathrm{DC} \times 160^{\circ}$ in the right eye and $-0.5 \mathrm{DS} /-$ $2.5 \mathrm{DC} \times 180^{\circ}$ in the left eye. Uncorrected visual acuities were 20/63 (corrected to 20/40), 20/32 and N10 in the right eye, 20/30 (corrected to 20/25), 20/32 and N8 in the right eye and 20/30, 20/32 and N8 with both eyes for distance, intermediate and near vision respectively. Uncorrected distance vision under night time simulating conditions (mesopic, low contrast) was 20/80 in the right eye, 20/50 in the left eye and 20/32 with both eyes. The patient was satisfied with his vision and reported no visual disturbances such as glare or haloes, but stated that he required spectacles for activities that require excellent far distance vision such as playing golf. Postoperative corneal topography and MTF could not be measured as the patient could not attend the appointment.

For patients that received the IC-8 IOLs (ie Cases 1-3), there was no significant difference $(\mathrm{P}>0.05)$ in the average MTF between the eyes that received the IC- 8 and the eyes

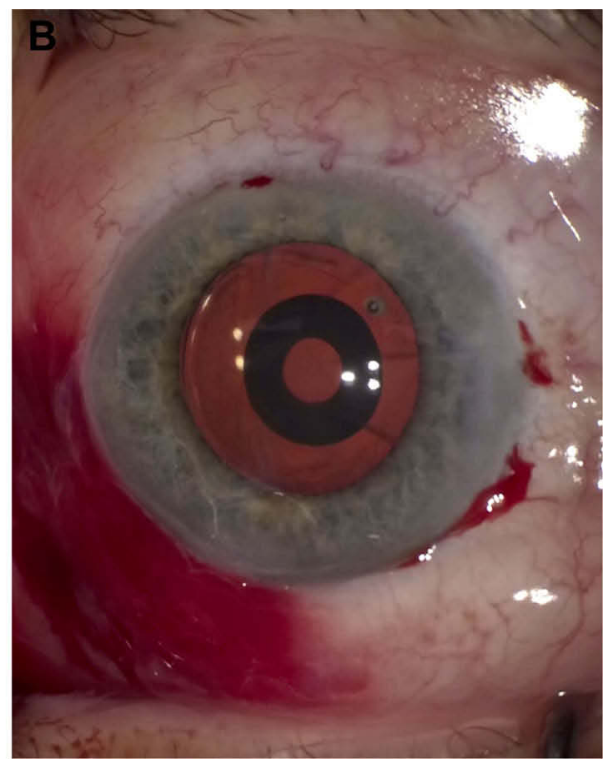

Figure 2 Intraoperative images of the left eye (A) prior to and (B) following implantation of the IC-8 IOL. 

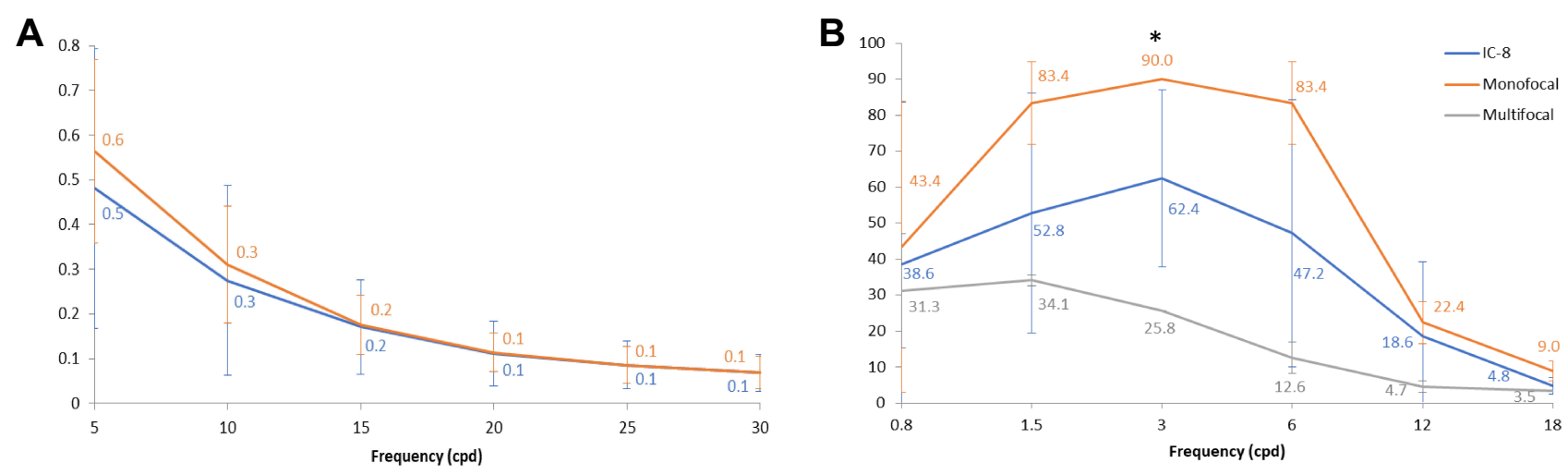

Figure 3 (A) Average automated MTF and (B) average uncorrected mesopic CSF in post-refractive patients following cataract surgery and lens implantation. Error bars are \pm I standard deviation. Significant differences $(P=0.05)$ are denoted by an asterisk.

that received the monofocal IOLs (Figure 3A). Similarly, there was no significant differences in average CSF between the eyes that received the IC- 8 , multifocal and monofocal IOLs $(\mathrm{P}>0.05)$, except for between eyes that received multifocal and monofocal IOLs at $3 \mathrm{cpd}$ $(\mathrm{P}<0.01)$, where the average CSF for the eyes that received the multifocal IOLs was reduced by $71 \%$ compared to the eyes that received the monofocal IOLs (Figure 3B).

\section{Discussion}

Cataract surgery in post-keratorefractive patients is complicated by inaccuracy in preoperative IOL measurements and high patient expectations. Multifocal IOLs have successfully restored good vision at all distances in patients with previous history of $\mathrm{RK}^{4,5}$ or LASIK. ${ }^{8,9}$ However, spherical IOLs can result in a reduction in postoperative $\mathrm{CS},{ }^{6,7}$ and visual disturbances such as glare or haloes which can negatively affect patient satisfaction. ${ }^{11,12}$ A recent clinical trial of 939M AT Lisa IOL implantation in eyes with virgin corneas reported high levels of patient satisfaction, with CS falling within the normal range, and a low incidence of visual disturbances. ${ }^{13}$ However, to our knowledge, no studies on the implantation of the AT Lisa 939M IOL in eyes with previous history of keratorefractive procedures have been published. The IC-8 IOL has recently provided patients with corneal irregularities resulting from trauma ${ }^{14}$ and previous kerato-refractive procedures ${ }^{15,16}$ an extended range of vision while avoiding the problems associated with monovision and multifocal IOLs; patients generally achieve spectacle independence and good vision at all distances, ${ }^{14-16}$ and CS and stereopsis ${ }^{16}$ are well maintained. This case series reports the outcomes of unilateral IC-8 IOL implantation in two patients with history of bilateral myopic RK and one patient with history of bilateral myopic RK and AK, and bilateral implantation of AT Lisa 939M trifocal toric IOLs in a single patient with history of PRK and LASIK.

Most of the complexity associated with lens implantation in post-RK eyes is due to the central corneal flattening that can lead to underestimation of the corneal power and to progressive hyperopic shift. To address this, the lens power was calculated using Barrett True $\mathrm{K}$ formula. This formula incorporates true net power into the calculation to accommodate for inconsistencies between central and peripheral corneal curvature. To counteract postoperative hyperopic shift, a slightly myopic target refraction of $-0.75 \mathrm{D}$ was used for eyes that were to receive the IC-8 IOL. Owing to the extended depth of focus of IC-8 IOL, the lens can tolerate up to $1.5 \mathrm{D}$ of cylindrical refractive error, ${ }^{17,18}$ accommodating for the residual refraction while offsetting any myopic shift. A target refraction of $0.0 \mathrm{D}$ to $-0.5 \mathrm{D}$ was used for the patient that received the AT Lisa IOLs and for eyes that received monofocal lenses. At 6 months postoperatively, all eyes that received the IC-8 IOL were within $\pm 0.5 \mathrm{D}$ of the target refraction of $-0.75 \mathrm{D}$ (ie postoperative spherical equivalents of -1.0 $\mathrm{D},-0.25 \mathrm{D}$ and $-0.875 \mathrm{D}$ for Case 1,2 and 3 respectively), improving from an average preoperative SE of $0.08 \mathrm{D}$ to a postoperative $\mathrm{SE}$ of $-0.71 \mathrm{D}$. All eyes that received the monofocal IOLs were also within $\pm 0.5 \mathrm{D}$ of the plano target, improving from $-0.875 \mathrm{D}$ preoperatively to $-0.125 \mathrm{D}$ postoperatively. Furthermore, binocular UDVA, UIVA and UNVA were $20 / 25$ or better, $20 / 16$ or better and N8 or better respectively, and two patients achieved full spectacle independence with the remaining patient requiring spectacles for small print only. Although 
there was up to $0.5 \mathrm{D}$ of refractive error despite the use of the Barrett True $\mathrm{K}$ formula to calculate lens power, it was well tolerated by all the three patients who received IC-8 IOL. All the patients expressed satisfaction with their vision and spectacle independence for most of the routine tasks. Notably, Cases 2 and 3 had -0.5 DC and -1.75 DC of against the rule residual astigmatism. Although this is usually poorly tolerated postoperatively, the pinhole effect of the IC-8 may allow for refractive error to be compensated for, and may in fact help to enhance depth of focus as induced astigmatism has previously been reported to help enhance depth of focus for near vision. ${ }^{19}$ The patient who received the AT Lisa IOLs (i.e., Case 4) presented postoperatively with fluctuating refraction, a common postoperative complication of RK, and postoperative myopia in both eyes; mean SE decreased from 1.875 $\mathrm{D}$ preoperatively to $-1.75 \mathrm{D}$ postoperatively. This overcorrection could be due to preoperative instability of the cornea and fluctuations in preoperative or postoperative refraction. Despite this, the patient was satisfied with his vision and did not express a need for glasses except when playing golf. As the post-refractive cornea can continue to get progressively flatter, longer term follow up will need to be performed to determine if these outcomes can be maintained.

Conventional monovision causes a loss in stereopsis that can negatively affect patient outcome. ${ }^{20}$ However, mini-monovision can yield similar stereoacuity to the normal population when combined with small aperture inlays. $^{20}$ The average postoperative stereopsis threshold for Case 1 and 2 was $300 \mathrm{sec}$ arc, within the normal range of the age-matched general population. ${ }^{21}$ However, Case 3 had reduced stereopsis (ie $>400 \mathrm{sec}$ arc) postoperatively. Postoperative SE for this patient was -0.875 in the IC- 8 eye and +0.5 in the monofocal eye, i.e., more than a dioptre difference possibly causing anisometropia and reduced stereopsis. This suggests that mini-monovision combined with small aperture technology can be well tolerated, however unintended postoperative anisometropia may still have a negative effect on stereoacuity.

Patients with previous history of corneal refractive procedures are susceptible to visual disturbances such as glare and haloes due to corneal irregularities and low tolerance. The pinhole effect of the IC-8 IOL may ameliorate the effect of peripheral HOAs, as there is a strong positive correlation between pupil size and the amount of HOAs following refractive procedures. ${ }^{22}$ Aspheric multifocal IOLs have also been reported to induce less HOAs than spherical IOLs, ${ }^{7}$ and the AT Lisa 939M IOL has been reported to have a low incidence of postoperative glare and haloes. ${ }^{13}$ No patients involved in this study reported visual disturbances, suggesting that corneal aberrations are well tolerated by these lenses.

Multifocal IOLs often result in poor CSdue to the splitting of light between the different diffraction zones of the lens. This is most pronounced under low light conditions and high spatial frequencies. ${ }^{23}$ CSwas tested using two methods; mesopic uncorrected CSF and automated MTF. CSF for eyes that received the multifocal IOLs was significantly reduced $(\mathrm{P}<0.05)$ compared to eyes that received the monofocal IOLs at $3 \mathrm{cpd}$ (Figure 3). However, the small sample size and inconsistency in patient characteristics make meaningful statistical analysis impossible. There was, however, a general trend of lower CSF in eyes with higher amounts of preoperative coma, i.e., more than approximately $0.2 \mu \mathrm{m}$. This is consistent with previous reports that mesopic CS in post-LASIK patients negatively correlates with coma-like aberrations when the photopic pupil is $<4 \mathrm{~mm}$, and with SAwhen the photopic pupil is $>4 \mathrm{~mm} \cdot{ }^{24,25}$ Differences in visual function between patients may therefore be dependent on variation in individual HOA profiles, and may suggest that visual function and CS may become compromised in some post-refractive patients with clinically significant HOAs that receive IC-8 or multifocal IOLs as previously reported., ${ }^{8,9}$ Although this may affect patient satisfaction, no patients for this study expressed dissatisfaction with their quality of vision, suggesting that reduced CS may be perceived as a fair trade off to achieve spectacle independence. A comprehensive large-scale study will need to be performed to fully evaluate how CS is affected by corneal HOAs, and how this effect may be tolerated by the IC-8 and AT Lisa 939M IOLs under different lighting conditions.

\section{Conclusion}

Current extended depth of focus IOLs can provide postrefractive patients with a good range of vision and high degree of spectacle independence. Patients who receive IC-8 IOLs however may require reading glasses under low light conditions when their visual function may be negatively affected, and patients that receive multifocal lenses such as the AT Lisa 939M IOL may experience reduced CS possibly due to the diffractive design of the IOL. Extended depth of focus lenses such as the IC-8, and diffractive multifocal lenses such as the AT Lisa 939M, may therefore be used in patients wanting spectacle independence following corneal refractive procedures but with caution and detailed preoperative counseling. 
Thorough analysis of the preoperative topography is essential and it is recommended that each patient is treated on a case by case basis rather than a one-size-fits-all approach.

\section{Abbreviations}

IOL, intraocular lens; RK, radial keratotomy; AK, astigmatic keratotomy; LASIK, laser-assisted in situ keratomileusis; $\mathrm{CS}$, contrast sensitivity; HOA, higher order aberration; SA, spherical aberration; VA, visual acuity; UDVA, uncorrected distance visual acuity; UIVA, uncorrected intermediate visual acuity, UNVA, uncorrected near visual acuity; CSF, contrast sensitivity function; MTF, modulation transfer function; CDVA, corrected distance visual acuity.

\section{Consent for Publication}

Written informed consent was obtained from all participants prior to testing.

\section{Data Sharing Statement}

Patient data is stored at Wollongong Eye Specialists and may be made available upon request.

\section{Author Contributions}

All authors contributed to data analysis, drafting or revising of article, gave final approval of the version to be published, and agree to be accountable for all aspects of the work.

\section{Disclosure}

The authors report no conflicts of interest in this work.

\section{References}

1. Messenger WB, Ambati BK. Unilateral implantation of a pseudoaccommodating toric intraocular lens in 2 patients after radial keratotomy. JCRS Online Case Rep. 2014;2:73-77. doi:10.1016/j. jcro.2014.09.005

2. Gupta I, Oakey Z, Ahmed F, et al. Spectacle independence after cataract extraction in post-radial keratotomy patients using hybrid monovision with ReSTOR multifocal and TECNIS monofocal intraocular lenses. Case Rep Ophthalmol. 2014;5:157-161. doi:10.1159/ 000363372

3. Chang JSM, Ng JCM, Chan VKC, et al. Visual outcomes, quality of vision, and quality of life of diffractive multifocal intraocular lens implantation after myopic laser in situ keratomileusis: a prospective, observational case series. J Ophthalmol. 2017;2017:645-540.

4. Nuzzi R, Monteu F, Tridico F. Implantation of a multifocal toric intraocular lens after radial keratotomy and cross-linking with hyperopia and astigmatism residues: a case report. Case Rep Ophthalmol. 2017;8:440-445. doi:10.1159/000479813

5. Kim KH, Seok K, Kim WS. Multifocal intraocular lens results in correcting presbyopia in eyes after radial keratotomy. Eye and Contact Lens. 2017;43:e22-e25. doi:10.1097/ICL.00000000000 00208
6. Kohnen T, Klaproth OK, Buhren J. Effect of intraocular lens asphericity on quality of vision after cataract removal: an intraindividual comparison. Ophthalmology. 2009;116:1697-1706. doi:10.1016/j. ophtha.2009.03.052

7. Tzelikis PF, Akaishi L, Trindade FC, et al. Spherical aberration and contrast sensitivity in eyes implanted with aspheric and spherical intraocular lenses: a comparative study. Am $J$ Ophthalmol. 2008;145:827-833. doi:10.1016/j.ajo.2007.12.023

8. Fernandez-vega L, Madrid-Costa D, Alfonso JF, et al. Optical and visual performance of diffractive intraocular lens implantation after myopic laser in situ keratomileusis. J Cataract Refract Surg. 2009;35:825-832. doi:10.1016/j.jcrs.2008.12.040

9. Alfonso JF, Madrid-Costa D, Poo-Lopez A, et al. Visual quality after diffractive intraocular lens implantation in eyes with previous myopic laser in situ keratomileusis. $J$ Cataract Refract Surg. 2008;34:1848-1854. doi:10.1016/j.jcrs.2008.07.023

10. Kinard K, Jarstad A, Olson RJ. Correlation of visual quality with satisfaction and function in a normal cohort of pseudophakic patients. $J$ Cataract Refract Surg. 2013;39:590-597. doi:10.1016/j.jcrs.2012.11.023

11. Khandelwal SS, Jun JJ, Make S, et al. Effectiveness of multifocal and monofocal intraocular lenses for cataract surgery and lens replacement: a systematic review and meta-analysis. Graef Arch Clin Exp. 2019;257:863-875. doi:10.1007/s00417-018-04218-6

12. de Silva SR, Evans JR, Kirthi V, et al. Multifocal versus monofocal intraocular lenses after cataract extraction. Cochrane Db Syst Rev. 2016;12:CD003169.

13. Piovella M, Colonval S, Kapp A, et al. Patient outcomes following implantation with a trifocal toric IOL: twelve-month prospective multicentre study. Eye. 2019;33:144-153. doi:10.1038/s41433-0180076-5

14. Schultz T, Dick HB. Small-aperture intraocular lens implantation in a patient with an irregular cornea. J Cataract Refract Surg. 2016;32:706-708. doi:10.3928/1081597X-20160721-01

15. Barnett V, Barsam A, Than J, et al. Small-aperture intraocular lens combined with secondary piggyback intraocular lens during cataract surgery after previous radial keratotomy. J Cataract Refract Surg. 2018;44:1042-1045. doi:10.1016/j.jcrs.2018.06.005

16. Agarwal S, Thornell EM. Cataract surgery with a small-aperture intraocular lens after previous corneal refractive surgery: visual outcomes and spectacle independence. $J$ Cataract Refract Surg. 2018;44:1150-1154. doi:10.1016/j.jcrs.2018.06.028

17. Dick HB, Piovella M, Vukich J, et al. Prospective multicentre trial of a small-aperture intraocular lens in cataract surgery. $J$ Cataract Refract Surg. 2017;43:956-968. doi:10.1016/j.jcrs.2017.04.038

18. Ang RE. Small-aperture intraocular lens tolerance to induced astigmatism. Clin Ophthalmol. 2018;12:1659-1664. doi:10.2147/ OPTH

19. Leube A, Ohlendorf A, Wahl S. The influence of induced astigmatism on the depth of focus. Optom Vis Sci. 2016;93:1228-1234. doi:10.1097/OPX.0000000000000961

20. Fernandez EJ, Schwarz C, Prieto PM, et al. Impact on stereo-acuity of two presbyopia correction approaches: monovision and small aperture inlay. Biomed Opt Express. 2013;4:822-830. doi:10.1364/ BOE.4.000822

21. Zaroff CM, Knutelska M, Frumkes TE. Variation in stereoacuity: normative description, fixation disparity, and the roles of aging and gender. Invest Ophthalmol Vis Sci. 2003;44:891-900. doi:10.1167/ iovs.02-0361

22. Martinez CE, Applegate RA, Klyce SD, et al. Effect of pupillary dilation on corneal optical aberrations afetr photorefractive keratectomy. Arch Ophthalmol. 1998;116:1053-1062. doi:10.1001/ archopht.116.8.1053

23. Montes-Mico R, Espana E, Bueno I, et al. Visual performance with multifocal lenses: mesopic contrast sensitivity under distance and near conditions. Ophthalmology. 2004;111:85-96. doi:10.1016/ S0161-6420(03)00862-5 
24. Oshika T, Tokunaga T, Samejima T, et al. Influence of pupil diametre on the relation between ocular higher-order aberration and contrast sensitivity after laser in situ keratomileusis. Cornea. 2006;47:1334-1338.
25. Zhao P, Li SM, L J, et al. Effects of higher-order aberrations on contrast sensitivity in normal eyes of a large myopic population. Int J Ophthalmol. 2017;10:1407-1411. doi:10.18240/ijo.2017.09.13

\section{Publish your work in this journal}

The International Medical Case Reports Journal is an international, peer-reviewed open-access journal publishing original case reports from all medical specialties. Previously unpublished medical posters are also accepted relating to any area of clinical or preclinical science. Submissions should not normally exceed 2,000 words or 4 published pages including figures, diagrams and references. The manuscript management system is completely online and includes a very quick and fair peer-review system, which is all easy to use. Visit http://www.dovepress.com/testimonials.php to read real quotes from published authors. 